\title{
Spin-orbit interaction in Hartree-Fock calculations
}

\author{
Ana R. Bautista \\ Departamento de Física Moderna, Universidad de Granada, \\ E-18071 Granada, Spain \\ Giampaolo Co' \\ Dipartimento di Fisica, Università di Lecce \\ and I.N.F.N. sezione di Lecce, I-73100 Lecce, Italy \\ Antonio M. Lallena \\ Departamento de Física Moderna, Universidad de Granada, \\ E-18071 Granada, Spain
}

\begin{abstract}
The contribution of the spin-orbit interaction in Hartree-Fock calculation for closed shell nuclei is studied. We obtain explicit expressions for the finite range spin-orbit force. New terms with respect to the traditional spin-orbit expressions are found. The importance of the finite-range is analyzed. Results obtained with spin-orbit terms taken from realistic interactions are presented. The effect of the spin-orbit isospin dependent terms is evaluated.
\end{abstract}

PACS 21.60.Jz 


\section{Introduction}

The success of the shell model in predicting the nuclear magic numbers is related to the presence of a strong spin-orbit term in the nuclear average potential. Few years after the formulation of the nuclear shell-model [1], evidences of spin-orbit terms in the nuclear interaction were identified [2] by analyzing polarized proton scattering data off complex nuclei [3].

The strong spin-orbit term of the nuclear average potential should be generated by an analogous term present in the nucleon-nucleon interaction. However, the connection between realistic nucleon-nucleon interactions, i.e. those built to reproduce the two nucleon scattering data and the deuteron properties, and the effective interactions, those used in nuclear structure effective theories, is still unclear.

In the present article we present a study on the relationship between the spin-orbit terms used in realistic nucleon-nucleon interaction and the analogous terms used in Hartree-Fock (HF) calculations. Our work has been done within the non relativistic framework where the spin-orbit terms can be easily isolated. In effect, in our HF calculations, we have used the explicit spin-orbit terms of the Argonne-Urbana nucleon-nucleon realistic potentials [4, 5, 6] describing, within the non relativistic framework, nucleon-nucleon elastic scattering data up to energies of about $300 \mathrm{MeV}$.

The spin-orbit interactions commonly used in HF calculations are of zero-range type and, in general, they are parametrized following the expressions proposed by Skyrme [7]. Also the Gogny interaction [8], which has a finite range for all the other channels, uses a Skyrme-like expression for the spin-orbit term.

We explicitly develop the expressions for a finite range spin-orbit interaction to be used in HF calculations. As expected, in addition to the direct term these expressions produce a contribution also in the Fock-Dirac exchange term of the HF equations. This term is not present when zero-range interactions are used. Also in the direct term there are some new contributions with respect to the expression obtained with the Skyrme interaction.

In the next paragraphs we present the detailed expressions of the HF equations when finite range spin-orbit terms are considered, then we discuss the importance of the finiterange and the effect of using spin-orbit terms taken from realistic nucleon-nucleon interactions. Finally we draw our conclusions.

\section{The formalism}

In ref. [9] we made an explicit presentation of the HF formalism with finite-range interactions. In the present article we extend the formalism in order to treat also the spin-orbit

terms. For this reason we recall here only those parts of the formalism involving the spin-orbit terms. 
The effective interaction used in our calculations has the form:

$$
V\left(\mathbf{r}_{1}, \mathbf{r}_{2}\right)=\sum_{p=1}^{8} V_{p}\left(\mathbf{r}_{1}, \mathbf{r}_{2}\right) O^{p}(1,2)
$$

where to the first 6 components used in ref. 9] we have added the spin-orbit terms defined as $O^{7}(1,2)=\mathbf{L} \cdot \mathbf{S}$ and $O^{8}(1,2)=\mathbf{L} \cdot \mathbf{S} \boldsymbol{\tau}_{1} \cdot \boldsymbol{\tau}_{2}$ with $\mathbf{L}=\left(\mathbf{r}_{1}-\mathbf{r}_{2}\right) \times\left(\mathbf{p}_{1}-\mathbf{p}_{2}\right)$ and $\mathbf{S}=\frac{1}{2}\left(\boldsymbol{\sigma}_{1}+\boldsymbol{\sigma}_{2}\right)$.

To take advantage of the spherical symmetry of the problem, we describe the single particle wave functions by separating the angular and the radial parts $\phi_{k}(\mathbf{r}) \equiv \tilde{k}(\Omega) u_{k}(r) / r$ where the subindex $k$ indicates all the quantum numbers necessary to identify the state and $\Omega$ the two angular coordinates $\theta$ and $\phi$.

The specific expression of the single particle wave functions allows us to reduce the HF equations into a set of differential equations of the type:

$$
-\frac{\hbar^{2}}{2 m_{k}}\left(\frac{\mathrm{d}^{2}}{\mathrm{~d} r^{2}}-\frac{l_{k}\left(l_{k}+1\right)}{r^{2}}\right) u_{k}(r)+U_{k}(r) u_{k}(r)-W_{k}(r)=\epsilon_{k} u_{k}(r),
$$

where we have defined:

$$
U_{k}(r)=\sum_{i} \int \mathrm{d} r^{\prime} u_{i}^{*}\left(r^{\prime}\right) \int \mathrm{d} \Omega \int \mathrm{d} \Omega^{\prime} \tilde{k}^{*}(\Omega) \tilde{i}^{*}\left(\Omega^{\prime}\right) V\left(\left|\mathbf{r}-\mathbf{r}^{\prime}\right|\right) \tilde{k}(\Omega) \tilde{i}\left(\Omega^{\prime}\right) u_{i}\left(r^{\prime}\right),
$$

and

$$
W_{k}(r)=\sum_{i} \int \mathrm{d} r^{\prime} u_{i}^{*}\left(r^{\prime}\right) \int \mathrm{d} \Omega \int \mathrm{d} \Omega^{\prime} \tilde{k}^{*}(\Omega) \tilde{i}^{*}\left(\Omega^{\prime}\right) V\left(\left|\mathbf{r}-\mathbf{r}^{\prime}\right|\right) \tilde{i}(\Omega) \tilde{k}\left(\Omega^{\prime}\right) u_{i}(r) u_{k}\left(r^{\prime}\right) .
$$

While the interaction depends from the relative distance between two nucleons, the HF equations (2) depend upon the distance of the particles from the origin of the reference system. The implementation of finite range interactions in the HF equations requires the separation of the coordinate variables in the interaction. For the central and tensor channels this separation is done by considering the interaction in coordinate space as Fourier transform of the interaction expressed in momentum space (see ref. [9] for details). For the spin-orbit channels $(p=7,8)$ we use a different strategy consisting in expanding in multipoles the interaction:

$$
V_{p}\left(r_{12}\right)=4 \pi \sum_{L M} \frac{1}{\widehat{L}^{2}} \mathcal{V}_{L}^{p}\left(r_{1}, r_{2}\right) Y_{L M}^{*}\left(\widehat{r}_{1}\right) Y_{L M}\left(\widehat{r}_{2}\right) .
$$

with $\widehat{L} \equiv \sqrt{2 L+1}$. ¿From the previous equation, making use of the orthogonality of the spherical harmonics, we obtain a close expression for the coefficients of the expansion:

$$
\mathcal{V}_{L}^{p}\left(r_{1}, r_{2}\right)=\frac{\widehat{L}^{2}}{2} \int_{-1}^{1} \mathrm{~d} \cos \theta_{12} V_{p}\left(r_{12}\right) P_{L}\left(\cos \theta_{12}\right)
$$

In the previous equation we have indicated with $P_{L}$ the Legendre polynomials and with $\theta_{12}$ the angle between $\mathbf{r}_{1}$ and $\mathbf{r}_{2}$. 
The details of the calculations of the spin-orbit matrix elements are given in the Appendix. We obtain for the direct term in the HF equations the following result:

$$
\left[U_{k}(r)\right]_{p=7,8}=2 \pi I_{k}^{p} \int \mathrm{d} r^{\prime} r^{\prime 2}\left\{\left[j_{k}\left(j_{k}+1\right)-l_{k}\left(l_{k}+1\right)-\frac{3}{4}\right] \mathcal{U}_{C}^{p}\left(r, r^{\prime}\right)+\mathcal{U}_{L S}^{p}\left(r, r^{\prime}\right)\right\}
$$

where

$$
I_{k}^{p}= \begin{cases}1, & p=7 \\ 2 t_{k}, & p=8\end{cases}
$$

and the potentials $\mathcal{U}_{C}^{p}$ and $\mathcal{U}_{L S}^{p}$ are given by:

$$
\mathcal{U}_{C}^{p}\left(r, r^{\prime}\right)=\left[\mathcal{V}_{0}^{p}\left(r, r^{\prime}\right)-\frac{1}{3} \frac{r^{\prime}}{r} \mathcal{V}_{1}^{p}\left(r, r^{\prime}\right)\right] \Omega_{C}^{p}\left(r^{\prime}\right), \quad p=7,8
$$

and

$$
\mathcal{U}_{L S}^{p}\left(r, r^{\prime}\right)=\left[\mathcal{V}_{0}^{p}\left(r, r^{\prime}\right)-\frac{1}{3} \frac{r}{r^{\prime}} \mathcal{V}_{1}^{p}\left(r, r^{\prime}\right)\right] \Omega_{L S}^{p}\left(r^{\prime}\right), \quad p=7,8
$$

The function $\Omega_{C}^{p}(r)$ used in the previous equations has been defined as:

$$
\Omega_{C}^{p}(r)= \begin{cases}\rho(r), & p=7 \\ \rho^{\pi}(r)-\rho^{\nu}(r), & p=8\end{cases}
$$

where $\rho^{\pi}(r)$ and $\rho^{\nu}(r)$ are the proton and neutron densities such as $\rho^{\pi}(r)+\rho^{\nu}(r)=\rho(r)$. The other function used in eq. (10) has been defined as:

$$
\Omega_{L S}^{p}(r)= \begin{cases}\rho_{L S}(r), & p=7 \\ \rho_{L S}^{\pi}(r)-\rho_{L S}^{\nu}(r), & p=8\end{cases}
$$

where $\rho_{L S}$ is the nucleon spin-density,

$$
\rho_{L S}(r)=\frac{1}{4 \pi} \sum_{i}\left[j_{i}\left(j_{i}+1\right)-l_{i}\left(l_{i}+1\right)-\frac{3}{4}\right] \widehat{j}_{i}^{2}\left(\frac{u_{i}(r)}{r}\right)^{2},
$$

and $\rho_{L S}^{\pi}(r)$ and $\rho_{L S}^{\nu}(r)$ are the analogous functions for protons and neutrons respectively.

For the exchange terms of eq. (2) we obtain:

$$
\left[W_{k}(r)\right]_{p=7,8}=\sum_{i L} I_{k i}^{p} \sum_{\alpha=1,5} \varepsilon_{k i L}^{(\alpha)} \int \mathrm{d} r^{\prime} \mathcal{W}_{k i L}^{p(\alpha)}\left(r, r^{\prime}\right),
$$

where

$$
I_{k i}^{p}= \begin{cases}\delta_{t_{k}, t_{i}}, & p=7 \\ 2 \delta_{t_{k},-t_{i}}+\delta_{t_{k}, t_{i}}, & p=8 .\end{cases}
$$

The new five functions $\varepsilon$ have been defined as:

$$
\varepsilon_{k i L}^{(\alpha)}=\sqrt{3}(-1)^{j_{i}+l_{i}+\frac{1}{2}} \widehat{l}_{k} \widehat{l}_{i} \widehat{j}_{i}^{2} \sum_{K}(-1)^{K} \widehat{K}^{2}\left(\begin{array}{ccc}
1 & K & L \\
1 & -1 & 0
\end{array}\right) \zeta_{k i}^{(\alpha)}(L, K), \quad \alpha=1, \ldots, 5,
$$


with $\zeta_{k i}^{(\alpha)}(L, K)$ given by:

$$
\begin{aligned}
\zeta_{k i}^{(1)}(L, K)= & \xi\left(l_{k}+l_{i}+L\right) \mathcal{T}_{k i}(L, K) \\
& {\left[\sqrt{l_{i}\left(l_{i}+1\right)}\left(\begin{array}{ccc}
l_{i} & l_{k} & K \\
-1 & 0 & 1
\end{array}\right)-\sqrt{l_{k}\left(l_{k}+1\right)}\left(\begin{array}{ccc}
l_{i} & l_{k} & K \\
0 & -1 & 1
\end{array}\right)\right] } \\
\zeta_{k i}^{(\alpha)}(L, K)= & \mathcal{G}_{k i}(L, K)\left\{\begin{array}{l}
(-2) \sqrt{l_{i}\left(l_{i}+1\right)}\left(\begin{array}{ccc}
l_{i} & l_{k} & K \\
-1 & 0 & 1
\end{array}\right)\left(\begin{array}{ccc}
1 & K & L \\
1 & -1 & 0
\end{array}\right), \\
2 \sqrt{l_{k}\left(l_{k}+1\right)}\left(\begin{array}{ccc}
l_{i} & l_{k} & K \\
0 & -1 & 1
\end{array}\right)\left(\begin{array}{ccc}
1 & K & L \\
1 & -1 & 0
\end{array}\right),
\end{array}\right. \\
(-\sqrt{2})\left(\begin{array}{ccc}
l_{i} & l_{k} & K \\
0 & 0 & 0
\end{array}\right)\left(\begin{array}{ccc}
1 & K & L \\
0 & 0 & 0
\end{array}\right), & \alpha=3
\end{aligned}
$$

In the previous expressions we have used the following definitions:

$$
\begin{aligned}
& \mathcal{T}_{k i}(L, K)=\left\{\begin{array}{ccc}
l_{k} & \frac{1}{2} & j_{k} \\
l_{i} & \frac{1}{2} & j_{i} \\
K & 1 & L
\end{array}\right\}\left(\begin{array}{ccc}
j_{k} & j_{i} & L \\
\frac{1}{2} & -\frac{1}{2} & 0
\end{array}\right) \\
&-\widehat{l}_{k} \widehat{l}_{i}\left\{\begin{array}{ccc}
l_{k} & \frac{1}{2} & j_{k} \\
l_{i} & \frac{1}{2} & j_{i} \\
L & 1 & K
\end{array}\right\}\left\{\begin{array}{ccc}
l_{k} & l_{i} & K \\
j_{i} & j_{k} & \frac{1}{2}
\end{array}\right\}\left(\begin{array}{ccc}
l_{k} & l_{i} & L \\
0 & 0 & 0
\end{array}\right), \\
& \mathcal{G}_{k i}(L, K)= \xi\left(l_{k}+l_{i}+L+1\right) \sum_{L^{\prime}} \xi\left(L+L^{\prime}+1\right) \widehat{L}^{\prime}\left(\begin{array}{ccc}
1 & K & L^{\prime} \\
1 & -1 & 0
\end{array}\right) \mathcal{T}_{k i}\left(L^{\prime}, K\right) .
\end{aligned}
$$

In the eqs. (14) we have used five new potentials:

$$
\begin{aligned}
& \mathcal{W}_{k i L}^{p(\alpha)}\left(r, r^{\prime}\right)=u_{i}^{*}\left(r^{\prime}\right) \mathcal{V}_{L}^{p}\left(r, r^{\prime}\right) u_{k}\left(r^{\prime}\right) u_{i}(r) \begin{cases}1, & \alpha=1, \\
\frac{r^{\prime}}{r}, & \alpha=2, \\
\frac{r}{r^{\prime}}, & \alpha=3,\end{cases} \\
& \mathcal{W}_{k i L}^{p(\alpha)}\left(r, r^{\prime}\right)= \begin{cases}r^{\prime} u_{i}^{*}\left(r^{\prime}\right) u_{k}\left(r^{\prime}\right) \mathcal{V}_{L}^{p}\left(r, r^{\prime}\right) \frac{\mathrm{d}}{\mathrm{d} r} u_{i}(r), \quad \alpha=4, \\
r u_{k}\left(r^{\prime}\right) \frac{\mathrm{d}}{\mathrm{d} r^{\prime}}\left[u_{i}^{*}\left(r^{\prime}\right) \mathcal{V}_{L}^{p}\left(r, r^{\prime}\right)\right] u_{i}(r), \quad \alpha=5 .\end{cases}
\end{aligned}
$$

Like in ref. [9] the numerical solution of eq. (2) has been obtained iteratively using the plane wave expansion method of refs. [10]. The center of mass motion has been considered in its simplest approximation, consisting in inserting the nucleon reduced mass in the hamiltonian. The single particle wave functions used to start the iterative procedure have been generated by a Saxon-Woods potential without spin-orbit and Coulomb terms. Therefore the starting wave functions for spin-orbit partners are the same. 


\section{Results}

In the same spirit of the work of ref. [9] we are more interested in investigating the validity of the commonly used approximations rather than proposing a new effective interaction to be used in HF calculations. This study has been conducted by adding different kinds of spin-orbit terms to a basic interaction composed by the four central terms of the force. These terms are described as a sum of two gaussians:

$$
V_{p}(r)=\sum_{i=1}^{2} A_{p i} \exp \left(-b_{i} r^{2}\right),
$$

with $p=1,2,3,4$. The parameters of this part of the interaction, which we call $B 1 a$, are compared in tab. 11 with the parameterization $B 1$ of Brink and Boeker [1]. The small differences are due to the fact that we have considered the Coulomb interaction and therefore we had to readjust the parameters of the force in order to reproduce the binding energy of ${ }^{4} \mathrm{He}$. We added to the $B 1 a$ interaction a finite range spin-orbit term of gaussian form:

$$
V_{7}\left(r_{12}\right)=A_{7} \exp \left(-b_{7} r_{12}^{2}\right)
$$

The finite range effects have been investigated by comparing the results obtained with the above interaction with those produced by adding to the $B 1 a$ force a zero range spin-orbit term of the form:

$$
V_{7}\left(r_{12}\right)=A_{7} \delta^{3}\left(\mathbf{r}_{1}-\mathbf{r}_{2}\right)
$$

The straightforward insertion of this expression in our formalism gives a contribution exactly equal to zero. The reason of this result can be traced back to the fact that we have developed our expressions using $\mathbf{L}=\left(\mathbf{r}_{1}-\mathbf{r}_{2}\right) \times\left(\mathbf{p}_{1}-\mathbf{p}_{2}\right)$. To get results different from zero for a zero-range spin-orbit interaction we set to zero the quantity $\mathcal{V}_{1}^{p}\left(r, r^{\prime}\right)$ in eqs. (9) and (10), and after inserting eq. (19) we obtained:

$$
\mathcal{U}_{C, L S}^{7}\left(r, r^{\prime}\right)=\frac{A_{7}}{2 r} \Omega_{C, L S}^{7}\left(r^{\prime}\right) \delta\left(r-r^{\prime}\right)
$$

The calculations done with this approach are labelled as $z$.

In addition to these effective interactions we have also used spin-orbit terms taken from microscopic forces: the Urbana V14 [4], Argonne V14 [5], and Argonne V18 [6] potentials. Our study has been restricted to the investigation of the doubly magic nuclei ${ }^{12} \mathrm{C},{ }^{16} \mathrm{O},{ }^{40} \mathrm{Ca},{ }^{48} \mathrm{Ca}$ and ${ }^{208} \mathrm{~Pb}$.

The finite range interaction (18) has been used to study the role played by the various terms of the spin-orbit potential. In a first set of calculations, labelled as $c$, only the $\mathcal{U}_{C}$ term of eq. (17) has been used. This is the only spin-orbit term present in shell-model calculations. In another set of calculations, denoted as $d$, we considered the full direct term, and, finally, the results identified with so have been obtained with all the spinorbit terms. These calculations have been done by changing every time the parameters of the force (18) to reproduce the $6.3 \mathrm{MeV}$ splitting between the protons 1p levels in ${ }^{16} \mathrm{O}$. The parameter $b_{7}$ was fixed to the arbitrary value of $1.2 \mathrm{fm}^{-2}$ and the fit of the 
splitting was obtained by changing $A_{7}$. The values of $A_{7}$ obtained in this way are, 108.75, -107.50, $-97.86 \mathrm{MeV}$ for the $c, d$, and so calculations respectively. The three interactions do not differ very much as it is shown in the panel I of the figure. This result indicates that the largest contribution from the spin-orbit force is coming from the $\mathcal{U}_{C}$ factor of the direct term. Since the other terms are small we have explored the possibility of avoiding their explicit calculation by simulating their effects with a readjustment of the force parameters. This is the reason why each type of calculation has been done with a different parametrization of the force, each of them reproducing the same empirical quantity.

In tab. 2 we compare the binding energies obtained with our calculations with the experimental ones [12]. In spite of the fact that we handle with a non-linear problem, the spin-orbit terms acts on the binding energies as expected. The main contribution to the binding energy is obtained by the sum of the single particle energies. In nuclei where all the spin-orbit partners are occupied the spin-orbit term lowers the energy of the $l+1 / 2$ level and increases that of the $l-1 / 2$ level, in such a way that the contribution to the nuclear binding energy is almost zero. In table 2 this is observed by looking at the values of the energies of ${ }^{16} \mathrm{O}$ and ${ }^{40} \mathrm{Ca}$ which are practically the same, independently from the spin-orbit force used. Clearly those nuclei where not all the spin-orbit partners are occupied are sensitive to the spin-orbit force, since the single particle energy of the last occupied level is lowered. The effect is seen in ${ }^{12} \mathrm{C},{ }^{48} \mathrm{Ca}$ and ${ }^{208} \mathrm{~Pb}$ where the binding energy increases, in absolute value, the stronger the spin-orbit force is.

The quantity most sensitive to the spin-orbit interaction is the energy splitting between spin-orbit partners levels. The energy splittings calculated for the various nuclei under investigation with the interactions proposed are compared in tabs. 3 and 4 with the Skyrme III [13] results and with the empirical values [14]. The experimental spectrum is more compressed than the theoretical one. This fact is well known [15], and it is related to the intrinsic limitations of the HF theory in the description of an interacting many-body system.

As expected, the splittings increase with increasing value of $l$. The splittings obtained with the zero range interaction $z$ become larger than those obtained with finite range interaction as the mass number of the nucleus increases. The results obtained with zerorange Skyrme interaction do not present this effect. In the Skyrme interaction there are velocity dependent terms generating spin-orbit like contributions which add to those produced by the genuine spin-orbit term. These velocity dependent terms simulate the effects of the finite range. We observe that the value of the splittings obtained with the Skyrme III interaction are comparable with those obtained with our finite range interactions.

¿From the comparison of the results of the $c$ and $d$ columns of tabs. 3 and 4 we infer information on the role of the terms $\mathcal{U}_{L S}^{p}$ in eq. (7). The inclusion of $\mathcal{U}_{L S}^{p}$ increases the splitting for all the nuclei considered but the magnitude of this increase is rather different for the various nuclei. We should not consider in our analysis the nucleus ${ }^{16} \mathrm{O}$ since it has been used to fit the interaction. We notice that the addition of $\mathcal{U}_{L S}^{p}$ produces quite small 
differences in the splitting of ${ }^{40} \mathrm{Ca}$ and ${ }^{208} \mathrm{~Pb}$ nuclei while they are remarkable in ${ }^{12} \mathrm{C}$ and ${ }^{48} \mathrm{Ca}$.

These results can be understood by considering that $\mathcal{U}_{L S}^{p}$ is related to the nuclear spin density, eq. (13). If we assume that the radial wave functions $u(r)$ are the same for spinorbit partners levels, the contribution of these two levels to the spin density is exactly zero. In real calculations these wave functions are slightly different, but the contribution to the spin density remains small. This explains the small increase of the splitting in ${ }^{40} \mathrm{Ca}$ and the relatively large modifications produced in ${ }^{12} \mathrm{C}$ and ${ }^{48} \mathrm{Ca}$. One should remark that only the unoccupied levels contribute to the spin density. For this reason the effect of $\mathcal{U}_{L S}^{p}$ is relatively large with respect to that of $\mathcal{U}_{C}^{p}$ in ${ }^{12} \mathrm{C}$ and ${ }^{48} \mathrm{Ca}$ where the number of single particle levels is relatively small. In a heavy nucleus like ${ }^{208} \mathrm{~Pb}$ there are many levels contributing in $\mathcal{U}_{C}^{p}$ and the effects of $\mathcal{U}_{L S}^{p}$ produced by a single level is relatively small.

The contribution of the exchange term can be seen by comparing the results of the $d$ and so columns. The variations with respect to the calculations done with only the direct terms can be as big as 10-15\%, but not all of them have the same sign. It seems that for all the $p$ states the splitting is reduced when the exchange term is considered, but it is increased in the $f, g$ and $h$ states. The situation for the $d$ states is even more complicated, since the splitting is reduced for the $1 d$ states in ${ }^{208} \mathrm{~Pb}$ but it has increased for all the other $d$ states. The contribution of the exchange term cannot be taken into account in calculations with the direct term only by modifying the force parameters.

The values of the splittings produced by the Urbana (U), and Argonne $\mathrm{V}_{18}$ interactions are comparable with those of our interactions, while the Argonne $V_{14}$ (A14) generates smaller values. The radial dependence of the spin-orbit terms of these interactions are shown in the panel II of the figure. It is remarkable that the results of $U$ and A18 are similar in spite of the large difference in the depth. The depth value of A14 is intermediate between those of the previous two forces, but its splittings are smaller. Th $U$ and A18 forces have similar range, while that of A14 is smaller. These facts indicate that our calculations are more sensitive to the range of the interaction than to its minimum value. In effect we recall that, in our calculations, a zero-range interaction does not produce any splitting.

The calculations done with the microscopic interactions include both spin-orbit and spin-orbit isospin terms. In order to study the importance of the isospin part of the spinorbit interaction we have repeated each calculation leaving out this terms. The differences of the results obtained with the full interaction and those without the isospin part are very small. In order to avoid a long list of numbers we give in tab. 5, for each nucleus under investigation, the minimum, the maximum and the average difference, in absolute value, between the calculated splittings. It appears clear the relatively small importance of this term of the interaction. This fact can be understood considering that the major contribution to the spin-orbit interaction is coming from the direct $\mathcal{U}_{C}^{p}$ term. For the spin-orbit isospin term of the interaction, the case $p=8$, the $\mathcal{U}_{C}^{p}$ term contains a function which is given by the difference between the proton and neutron density distributions, eq. (11). In all the nuclei we have considered this difference is small and particularly small 
in those nuclei having the same number of protons and neutron $\left({ }^{16} \mathrm{O}\right.$ and $\left.{ }^{40} \mathrm{Ca}\right)$. In effect the maximum differences are larger in ${ }^{48} \mathrm{Ca}$ and ${ }^{208} \mathrm{~Pb}$ than in ${ }^{16} \mathrm{O}$ and ${ }^{40} \mathrm{Ca}$.

\section{Summary and Conclusions}

In this article we have presented a formalism to treat finite range spin-orbit interactions in HF calculations. The finite range of the interaction generates additional terms with respect to the usual shell model expression. One of these is the contribution to the exchange Fock-Dirac term in the HF equation (2). Also in the direct (Hartree) term of this equation there is a new part: the $\mathcal{U}_{L S}$ piece of eq. (7). The major goal of our work was the investigation of the effects produced by these new components. This has been done by adding different type of spin-orbit terms to a fixed interaction active only in the four central channels. We have used a spin-orbit interaction of a gaussian form whose parameters have been fixed to reproduce the energy splitting of the proton $1 p$ levels in ${ }^{16} \mathrm{O}$.

We have shown that the largest part of the spin-orbit effects in HF calculations is produced by the traditional shell model term, $\mathcal{U}_{C}$ in eq. (7). The contribution of the other term, $\mathcal{U}_{L S}$, is very small and it can be simulated by a redefinition of the parameters of the force. The role of the exchange term is more complicated: its inclusion in the calculations modifies by a maximum of $15 \%$ the values of the spin-orbit splittings. The complication arises because these modifications do not have the same sign for all the nuclei studied. In calculations done with only the direct terms, it is not possible to simulate the exchange effects by simply readjusting the parameters of the interaction.

Forcing our formalism to handle zero-range spin-orbit interactions we have studied, by comparison, the importance of the finite range. We found that calculations done with zero-range interaction produce energy splittings which, in heavy nuclei, are much larger than the empirical ones. Traditional HF calculations use spin-orbit zero-range terms of Skyrme type [7]. These expressions produce contributions to the hamiltonian which are related to the derivative of the density distribution, while our expressions depend directly from the density distribution. The dependence from the derivative of the density distribution simulate effects produced by the finite range of the force.

We have done calculations with spin-orbit terms taken from microscopic interactions [4, 5, 6] and we have obtained splittings close to those produced by our effective spinorbit interactions. This would indicate that the medium does not affect the spin-orbit term of the realistic interaction, in agreement with the findings of G-matrix calculations [16]. We would like to point out, however, that the observables we have investigated are more sensitive to the global properties of the spin-orbit potential than to its details.

Modifications of the local properties of the interaction would not produce effects on our results.

We have also investigated the effects of the spin-orbit isospin term of the interaction, and we found them to be very small. These terms are related to the differences between 
protons and neutrons density and spin-orbit density distributions. In our calculations these quantities are rather small even for a nucleus with a large neutron excess like ${ }^{208} \mathrm{~Pb}$. There are however indications for observables which seems to be sensitive to this part of the potential [17.

The comparison of the results of our calculations with the empirical values of the splittings on the various nuclei investigated is not satisfactory. The empirical splittings are smaller than those we have obtained, except for ${ }^{12} \mathrm{C}$. This is a well known problem of the HF theory, and it could be solved only by using theories going beyond the mean field description of the nucleus.

\section{Appendix}

Since we have developed the HF equations in spherical coordinates it is necessary to express the operator $\mathbf{L} \cdot \mathbf{S}$ in terms of these coordinates. For this purpose we define an operator $O(i j k)$ as:

$$
O(i j k) \equiv(-1)^{i+j} \mathbf{r}_{i} \times \mathbf{p}_{j} \cdot \mathbf{s}_{k}=\sqrt{\frac{2 \pi}{3}}(-1)^{i+j} r_{i} \sum_{\mu}(-1)^{1-\mu} Y_{1 \mu}\left(\widehat{r}_{i}\right)\left[\nabla_{j} \otimes \sigma(k)\right]_{-\mu}^{1},
$$

where we have set $\hbar=1$ and the indexes $i, j, k$ can assume only two values, 1 and 2 for example. The previous formula has been obtained by expressing $r$ in terms of spherical harmonics and by making explicit use of the tensor product properties. Using the above operator we can write the spin-orbit channels of the force as:

$$
\begin{aligned}
V_{p}\left(r_{12}\right) O_{p}(1,2) \equiv & V_{p}\left(r_{12}\right) \mathcal{I}^{p} \sum_{i, j, k=1,2} O(i j k) \\
= & 4 \pi \sqrt{\frac{2 \pi}{3}} \mathcal{I}^{p} \sum_{L} \frac{1}{\widehat{L}^{2}} \mathcal{V}_{L}^{p}\left(r_{1}, r_{2}\right) \sum_{j k}(-1)^{j} \sum_{\mu}(-1)^{1-\mu}\left[\nabla_{j} \otimes \sigma(k)\right]_{-\mu}^{1} \\
& \sum_{i M}(-1)^{M+i} r_{i} Y_{L-M}\left(\widehat{r}_{1}\right) Y_{L M}\left(\widehat{r}_{2}\right) Y_{1 \mu}\left(\widehat{r}_{i}\right), \quad p=7,8
\end{aligned}
$$

The operator $\mathcal{I}^{p}$ has been defined as:

$$
\mathcal{I}^{p}= \begin{cases}1, & p=7 \\ \boldsymbol{\tau}(1) \cdot \boldsymbol{\tau}(2), & p=8\end{cases}
$$

In eq. (22) we make the sum on $M$ e $\mu$, and we obtain a more synthetic expression:

$$
\begin{aligned}
V_{p}\left(r_{12}\right) O_{p}(1,2)= & -2 \sqrt{2 \pi} \mathcal{I}^{p} \sum_{L L^{\prime}} \frac{\widehat{L^{\prime}}}{\widehat{L}}\left(\begin{array}{ccc}
L & L^{\prime} & 1 \\
0 & 0 & 0
\end{array}\right) \mathcal{V}_{L}^{p}\left(r_{1}, r_{2}\right) \\
& \sum_{i j k} O_{00}^{L L^{\prime}}(i j k), \quad p=7,8,
\end{aligned}
$$


where we have defined new set of operators as:

$$
O_{00}^{L L^{\prime}}(i j k)=(-1)^{i+j} r_{i}\left[\left[Y_{L^{\prime}}\left(\widehat{r}_{i}\right) \otimes Y_{L}\left(\widehat{r}_{\dot{\imath}}\right)\right]^{1} \otimes\left[\nabla_{j} \otimes \sigma(k)\right]^{1}\right]_{0}^{0}
$$

and where we have defined:

$$
\mathbf{r}_{\dot{\psi}}=\left\{\begin{array}{lll}
\mathbf{r}_{1}, & \text { for } & i=2 \\
\mathbf{r}_{2}, & \text { for } & i=1
\end{array}\right.
$$

It is convenient to express these operators such as the coordinates of each particle are separated:

$$
O_{00}^{L L^{\prime}}(i j k)=\sqrt{3} \sum_{K M}(-1)^{L+M}\left\{\begin{array}{ccc}
L & L^{\prime} & 1 \\
1 & 1 & K
\end{array}\right\} \tilde{O}_{L L^{\prime} K}^{M}(i j k)
$$

where we have used the Racah $6-j$ symbol and we have defined the operators:

$$
\tilde{O}_{L L^{\prime} K}^{M}(i j k)=e(i j k) \bar{A}_{J M}^{(i j k)}(1) \bar{B}_{J-M}^{(i j k)}(2) .
$$

The expressions of the three terms of the above equation, for each value of $(i j k)$ are given in tab. 6 as functions of the following operators:

$$
\begin{aligned}
\mathcal{C}_{\lambda \mu}^{L}(i) & =\left[Y_{L}\left(\widehat{r}_{i}\right) \otimes \nabla_{i}\right]_{\mu}^{\lambda} \\
\mathcal{S}_{\lambda \mu}^{L K}(i) & =\left[\left[Y_{L}\left(\widehat{r}_{i}\right) \otimes \nabla_{i}\right]^{K} \otimes \sigma(i)\right]_{\mu}^{\lambda} \equiv\left[\mathcal{C}_{K}^{L}(i) \otimes \sigma(i)\right]_{\mu}^{\lambda} \\
\mathcal{M}_{\lambda \mu}^{L}(i) & =\left[Y_{L}\left(\widehat{r}_{i}\right) \otimes \sigma(i)\right]_{\mu}^{\lambda} .
\end{aligned}
$$

At the end we express the spin-orbit terms of the interaction as:

$$
\begin{aligned}
V_{p}\left(r_{12}\right) O_{p}(1,2)= & 4 \pi \mathcal{I}^{p} \sum_{L L^{\prime} K} f\left(L, L^{\prime}, K\right) \mathcal{V}_{L}^{p}\left(r_{1}, r_{2}\right) \\
& \sum_{i j k} \sum_{M}(-1)^{M} \tilde{O}_{L L^{\prime} K}^{M}(i j k), \quad p=7,8,
\end{aligned}
$$

where $f$ is given by:

$$
f\left(L, L^{\prime}, K\right)=(-)^{L+K} \xi\left(L+L^{\prime}+1\right) \frac{\widehat{L^{\prime}}}{\widehat{L}}\left(\begin{array}{ccc}
1 & K & L^{\prime} \\
1 & -1 & 0
\end{array}\right)\left(\begin{array}{ccc}
1 & K & L \\
1 & -1 & 0
\end{array}\right) .
$$

In the calculation of the HF equations for the spin-orbit channels we have used the results corresponding to the matrix elements $\tilde{O}_{L L^{\prime} K}^{M}(i j k)$ which in the tab. 6 are shown to be function of $\mathcal{C}_{L M}^{K}, \mathcal{M}_{L M}^{K}, \mathcal{S}_{L M}^{L^{\prime} K}$ defined in (29) and of the spherical harmonics $Y_{L M}(\widehat{r})$.

Using the function $\xi(l)=1$ if $l$ is even and $=0$ if $l$ is odd, we express the reduced matrix elements for the spherical harmonics as:

$$
\left\langle l \frac{1}{2} j \| Y_{L}|| l^{\prime} \frac{1}{2} j^{\prime}\right\rangle=\frac{1}{\sqrt{4 \pi}}(-1)^{j^{\prime}+L+\frac{3}{2}} \widehat{j} \widehat{j^{\prime}} \widehat{L} \xi\left(l+l^{\prime}+L\right)\left(\begin{array}{ccc}
j & j^{\prime} & L \\
\frac{1}{2} & -\frac{1}{2} & 0
\end{array}\right)
$$


For the other three operators we obtain the following operators:

$$
\begin{aligned}
& \left\langle l \frac{1}{2} j|| \mathcal{S}_{L}^{L^{\prime} K}|| l^{\prime} \frac{1}{2} j^{\prime}\right\rangle=\sqrt{\frac{3}{2 \pi}}(-1)^{l^{\prime}} \widehat{j} \hat{j^{\prime}} \widehat{l} \widehat{l} l^{\prime} \widehat{L} \widehat{L^{\prime}} \widehat{K}\left\{\begin{array}{ccc}
l & \frac{1}{2} & j \\
l^{\prime} & \frac{1}{2} & j^{\prime} \\
K & 1 & L
\end{array}\right\} \\
& \left\{\sqrt{2} \xi\left(l+l^{\prime}+L^{\prime}+1\right)\left(\begin{array}{ccc}
l^{\prime} & K & l \\
1 & -1 & 0
\end{array}\right)\left(\begin{array}{ccc}
K & 1 & L^{\prime} \\
1 & -1 & 0
\end{array}\right) \frac{\sqrt{l^{\prime}\left(l^{\prime}+1\right)}}{r}\right. \\
& \left.+\left(\begin{array}{ccc}
l^{\prime} & K & l \\
0 & 0 & 0
\end{array}\right)\left(\begin{array}{ccc}
K & 1 & L^{\prime} \\
0 & 0 & 0
\end{array}\right) \frac{\mathrm{d}}{\mathrm{d} r}\right\} \\
& \left\langle l \frac{1}{2} j\left\|\mathcal{C}_{L}^{K}\right\| l^{\prime} \frac{1}{2} j^{\prime}\right\rangle=\frac{1}{\sqrt{4 \pi}}(-1)^{l+l^{\prime}+\frac{1}{2}+j^{\prime}+L} \widehat{j} \widehat{j^{\prime}} \widehat{l} \widehat{l^{\prime}} \widehat{L} \widehat{K}\left\{\begin{array}{ccc}
l & j & \frac{1}{2} \\
j^{\prime} & l^{\prime} & L
\end{array}\right\} \\
& \left\{\sqrt{2} \xi\left(l+l^{\prime}+K+1\right)\left(\begin{array}{ccc}
l^{\prime} & L & l \\
1 & -1 & 0
\end{array}\right)\left(\begin{array}{ccc}
L & 1 & K \\
1 & -1 & 0
\end{array}\right) \frac{\sqrt{l^{\prime}\left(l^{\prime}+1\right)}}{r}\right. \\
& \left.+\left(\begin{array}{ccc}
l^{\prime} & L & l \\
0 & 0 & 0
\end{array}\right)\left(\begin{array}{ccc}
L & 1 & K \\
0 & 0 & 0
\end{array}\right) \frac{\mathrm{d}}{\mathrm{d} r}\right\} \\
& \left\langle l \frac{1}{2} j|| \mathcal{M}_{L}^{K} \| l^{\prime} \frac{1}{2} j^{\prime}\right\rangle=\sqrt{\frac{3}{2 \pi}}(-1)^{l} \widehat{j} \hat{j}^{\prime} \widehat{l} \widehat{l} l^{\prime} \widehat{L} \widehat{K}\left\{\begin{array}{ccc}
l & \frac{1}{2} & j \\
l^{\prime} & \frac{1}{2} & j^{\prime} \\
K & 1 & L
\end{array}\right\}\left(\begin{array}{ccc}
l & K & l^{\prime} \\
0 & 0 & 0
\end{array}\right) .
\end{aligned}
$$

\section{References}

[1] Feenberg E., Phys. Rev., 75 (1949) 320; Haxel O., Jensen H.D., Suess H.E., Phys. Rev., 75 (1949) 1766; Mayer M.G., Phys. Rev., 75 (1949) 1969; Mayer M.G., Phys. Rev., 78 (1950) 22;

[2] Fermi E., Nuov. Cim. 11 (1954) 407.

[3] Hensinkfeld M., Freier G.D., Phys. Rev., 85 (1952) 80.

[4] Lagaris I.E. and Pandharipande V.R., Nucl. Phys. A, 359 (1981) 331.

[5] Wiringa R.B., Smith R.A. and Ainsworth T.L., Phys. Rev. C, 29 (1984) 1207.

[6] Wiringa R.B., Stoks V.G.J. and Schiavilla R., Phys. Rev. C, 51 (1995) 38;

[7] Bell J.S., Skyrme T.H., Phil. Mag. 1 (1956) 1055; Skyrme T.H., Nucl. Phys. 9 (1959) 641.

[8] Dechargè J., Gogny D., Phys. Rev. C 21 (1980) 1568.

[9] Co' G., Lallena A.M., Nuov. Cim. 111A (1998) 527. 
[10] Guardiola R., Ros J., Jour. Comp. Phys., 45 (1982) 374; Jour. Comp. Phys., 45 (1982) 390; Guardiola R., Schneider H., Ros J., Anales de Física A, 78 (1982) 154.

[11] Brink D.M., Boecker E., Nucl. Phys. A, 91 (1967) 1.

[12] Audi G., Wapstra A.H., Nucl. Phys. A, 565 (1993) 1.

[13] Beiner M, Flocard H., Van Giai N., Quentin P., Nucl. Phys. A, 238, (1975) 29.

[14] Campi X., Sprung D.W., Nucl. Phys. A, 194 (1972) 401.

[15] Ring P., Schuck P., The nuclear many body problem (Springer-Verlag, New York) 1980,p. 214-215.

[16] Nakayama K., Krewald S., Speth J., Love G., Nucl. Phys., A431 (1984) 419.

[17] Lalazissis G.A., Vretenar D., Pöschl W., Ring P., Phys. Lett. B 418 (1998) 7. 
Table 1: Parameters of the central force $B 1 a$ compared with the original parameterization of the Brink and Boeker $B 1$ force [11]. The values of the $A$ are expressed in $\mathrm{MeV}$, while those of $b$ in $\mathrm{fm}^{-2}$.

\begin{tabular}{crr}
\hline & $B 1$ & $B 1 a$ \\
\hline$A_{11}$ & -55.12 & -61.51 \\
$A_{12}$ & 647.06 & 647.06 \\
$A_{p 1}$ & 17.10 & 15.82 \\
$A_{p 2}$ & 51.51 & 51.51 \\
$b_{1}$ & 0.51 & 0.51 \\
$b_{2}$ & 2.04 & 2.04 \\
\hline
\end{tabular}

Table 2: Binding energies, in $\mathrm{MeV}$, for the nuclei investigated. The force $B 1 a$ does not have spin-orbit terms, the $z, c, d$ and so calculations are discussed in the text. The interactions labeled $U, A 14$ and $A 18$ have been obtained by adding to $B 1 a$ the spin-orbit terms of the Urbana V14 potential [司], of the Argonne V14 [0] potential and the Argonne V18 [6] potential. The experimental energies are taken from ref. [12].

\begin{tabular}{cccccc}
\hline \multicolumn{1}{c}{${ }^{12} \mathrm{C}$} & ${ }^{16} \mathrm{O}$ & ${ }^{40} \mathrm{Ca}$ & ${ }^{48} \mathrm{Ca}$ & ${ }^{208} \mathrm{~Pb}$ \\
\hline$B 1 a$ & -48.89 & -116.09 & -341.54 & -380.48 & -1854.00 \\
$z$ & -54.47 & -116.08 & -341.43 & -402.67 & -2053.15 \\
$c$ & -55.26 & -116.09 & -341.52 & -397.74 & -1913.47 \\
$d$ & -63.13 & -116.42 & -342.30 & -413.36 & -1985.81 \\
$s o$ & -67.37 & -116.42 & -342.24 & -420.60 & -2007.25 \\
$U$ & -62.77 & -116.46 & -342.27 & -411.75 & -1969.91 \\
$A 14$ & -55.18 & -116.19 & -341.72 & -395.20 & -1907.95 \\
$A 18$ & -61.20 & -116.37 & -342.10 & -408.55 & -1960.31 \\
$\exp$ & -92.16 & -127.62 & -342.05 & -416.00 & -1636.45 \\
\hline
\end{tabular}


Table 3: Spin-orbit splittings in MeV for the nuclei investigated. The empirical values have been calculated using the compilation of ref. [14. $\pi$ stands for protons, $\nu$ for neutrons.

\begin{tabular}{cccccccccc}
\hline & $z$ & $c$ & $d$ & $s o$ & $\mathrm{U}$ & $\mathrm{A} 14$ & A18 & SIII & exp \\
\hline${ }^{12} \mathrm{C}$ & & & & & & & & & \\
$1 \mathrm{p} \pi$ & 3.37 & 3.90 & 4.97 & 5.76 & 4.88 & 1.58 & 4.16 & & 14.00 \\
$1 \mathrm{p} \nu$ & 3.05 & 3.57 & 4.65 & 5.46 & 4.61 & 1.24 & 3.83 & & 13.76 \\
\hline${ }^{16} \mathrm{O}$ & & & & & & & & & \\
$1 \mathrm{p} \pi$ & 6.30 & 6.31 & 6.31 & 6.32 & 5.78 & 2.86 & 5.33 & 5.90 & 6.33 \\
$1 \mathrm{p} \nu$ & 6.38 & 6.36 & 6.36 & 6.37 & 5.89 & 2.91 & 5.41 & 6.03 & 6.16 \\
\hline${ }^{40} \mathrm{Ca}$ & & & & & & & & & \\
$1 \mathrm{p} \pi$ & 9.38 & 5.56 & 5.64 & 5.35 & 4.91 & 2.40 & 4.59 & 3.31 & \\
$1 \mathrm{~d} \pi$ & 12.14 & 8.36 & 8.45 & 9.16 & 7.52 & 3.70 & 6.99 & 6.18 & 7.17 \\
$1 \mathrm{p} \nu$ & 9.47 & 5.58 & 5.66 & 5.38 & 5.03 & 2.47 & 4.67 & 3.38 & 3.00 \\
$1 \mathrm{~d} \nu$ & 12.43 & 8.46 & 8.56 & 9.30 & 7.74 & 3.82 & 7.15 & 6.33 & 6.30 \\
\hline${ }^{48} \mathrm{Ca}$ & & & & & & & & & \\
$1 \mathrm{p} \pi$ & 10.05 & 4.62 & 5.11 & 4.94 & 4.47 & 2.10 & 4.13 & 2.72 & \\
$1 \mathrm{~d} \pi$ & 13.97 & 7.50 & 8.27 & 9.03 & 7.52 & 3.70 & 6.99 & 6.18 & 4.30 \\
$1 \mathrm{p} \nu$ & 9.25 & 3.89 & 4.35 & 3.76 & 3.41 & 1.23 & 3.20 & 2.36 & \\
$1 \mathrm{~d} \nu$ & 12.68 & 6.35 & 7.09 & 7.49 & 5.98 & 2.21 & 5.47 & 5.50 & 3.60 \\
$1 \mathrm{f} \nu$ & 11.69 & 7.82 & 8.53 & 10.17 & 8.07 & 3.28 & 7.16 & & 8.74 \\
\hline
\end{tabular}


Table 4: The same as tab. 3 for ${ }^{208} \mathrm{~Pb}$.

\begin{tabular}{cccccccccc}
\hline & $z$ & $c$ & $d$ & $s o$ & $\mathrm{U}$ & A14 & A18 & SIII & exp \\
\hline${ }^{208} \mathrm{~Pb}$ & & & & & & & & & \\
$1 \mathrm{p} \pi$ & 14.61 & 1.98 & 2.05 & 1.60 & 1.61 & 0.67 & 1.54 & 0.52 & \\
$1 \mathrm{~d} \pi$ & 22.32 & 3.36 & 3.63 & 3.56 & 2.79 & 1.08 & 2.66 & 1.25 & \\
$1 \mathrm{f} \pi$ & 28.47 & 5.01 & 5.54 & 5.86 & 4.33 & 1.67 & 4.10 & 2.34 & \\
$1 \mathrm{~g} \pi$ & 32.76 & 6.97 & 7.77 & 8.56 & 6.26 & 2.53 & 5.88 & 3.76 & \\
$1 \mathrm{~h} \pi$ & 34.31 & 9.15 & 10.17 & 11.47 & & & & & 5.57 \\
$2 \mathrm{p} \pi$ & 12.55 & 2.34 & 2.45 & 1.96 & 2.12 & 0.91 & 1.96 & 0.99 & \\
$2 \mathrm{~d} \pi$ & 18.78 & 4.00 & 4.18 & 4.29 & 3.60 & 1.63 & 3.36 & 1.76 & 1.32 \\
$2 \mathrm{f} \pi$ & 21.03 & 5.23 & 5.46 & 5.98 & 4.77 & 2.00 & 4.41 & 2.35 & 1.92 \\
$1 \mathrm{p} \nu$ & 14.63 & 2.05 & 2.11 & 1.46 & 1.80 & 0.83 & 1.68 & 0.53 & \\
$1 \mathrm{~d} \nu$ & 22.44 & 3.48 & 3.74 & 3.55 & 3.17 & 1.38 & 2.94 & 1.26 & \\
$1 \mathrm{f} \nu$ & 28.63 & 5.10 & 5.64 & 5.83 & 4.77 & 2.00 & 4.41 & 2.35 & \\
$1 \mathrm{~g} \nu$ & 32.85 & 6.96 & 7.77 & 8.38 & 6.67 & 2.79 & 6.13 & 3.81 & \\
$1 \mathrm{~h} \nu$ & 34.06 & 8.89 & 9.92 & 11.08 & 8.79 & 3.75 & 8.00 & 5.56 & \\
$2 \mathrm{p} \nu$ & 12.50 & 2.31 & 2.43 & 1.58 & 2.08 & 0.89 & 1.93 & 1.08 & \\
$2 \mathrm{~d} \nu$ & 18.74 & 3.92 & 4.10 & 3.96 & 3.73 & 1.68 & 3.40 & 1.98 & \\
$2 \mathrm{f} \nu$ & 20.94 & 5.15 & 5.36 & 5.97 & 5.17 & 2.41 & 4.64 & 2.78 & 1.77 \\
$3 \mathrm{p} \nu$ & 8.05 & 2.01 & 2.01 & 1.52 & 1.95 & 0.92 & 1.76 & 1.03 & 0.94 \\
\hline
\end{tabular}

Table 5: Differences in $\mathrm{MeV}$ between the splittings presented in tabs. 3 and $\mathrm{E}$ and those obtained leaving out the isospin part of the spin-orbit interaction for Urbana, Argonne V14 and Argonne V18 potentials. We give here the minimum, maximum and average difference, in absolute value.

\begin{tabular}{cccc}
\hline & Minimum & Maximum & Average \\
\hline${ }^{16} \mathrm{O}$ & 0.010 & 0.030 & 0.018 \\
${ }^{40} \mathrm{Ca}$ & 0.000 & 0.070 & 0.032 \\
${ }^{48} \mathrm{Ca}$ & 0.020 & 0.290 & 0.088 \\
${ }^{208} \mathrm{~Pb}$ & 0.000 & 0.450 & 0.128 \\
\hline
\end{tabular}


Table 6: Expressions of the $\tilde{O}_{L L^{\prime} K}^{M}(i j k)$ operators.

\begin{tabular}{|c|c|c|c|}
\hline$i j k$ & $e(i j k)$ & $\bar{A}_{J M}^{(i j k)}$ & $\bar{B}_{J-M}^{(i j k)}$ \\
\hline 111 & $-r_{1} \frac{\widehat{K}}{\widehat{L}}$ & $\mathcal{S}_{L M}^{L^{\prime} K}$ & $Y_{L-M}$ \\
\hline 112 & $r_{1}$ & $\mathcal{C}_{K M}^{L^{\prime}}$ & $\mathcal{M}_{K-M}^{L}$ \\
\hline 121 & $r_{1}$ & $\mathcal{M}_{K M}^{L^{\prime}}$ & $\mathcal{C}_{K-M}^{L}$ \\
\hline 122 & $-r_{1} \frac{\widehat{K}}{\bar{L}^{\prime}}$ & $Y_{L^{\prime} M}$ & $\mathcal{S}_{L^{\prime}-M}^{L K}$ \\
\hline 211 & $-r_{2} \frac{\widehat{K}}{\widehat{L^{\prime}}}$ & $\mathcal{S}_{L^{\prime} M}^{L K}$ & $Y_{L^{\prime}-M}$ \\
\hline 212 & $r_{2}$ & $\mathcal{C}_{K M}^{L}$ & $\mathcal{M}_{K-M}^{L^{\prime}}$ \\
\hline 221 & $r_{2}$ & $\mathcal{M}_{K M}^{L}$ & $\mathcal{C}_{K-M}^{L^{\prime}}$ \\
\hline 222 & $-r_{2} \frac{\widehat{K}}{\widehat{L}}$ & $Y_{L M}$ & $\mathcal{S}_{L-M}^{L^{\prime} K}$ \\
\hline
\end{tabular}



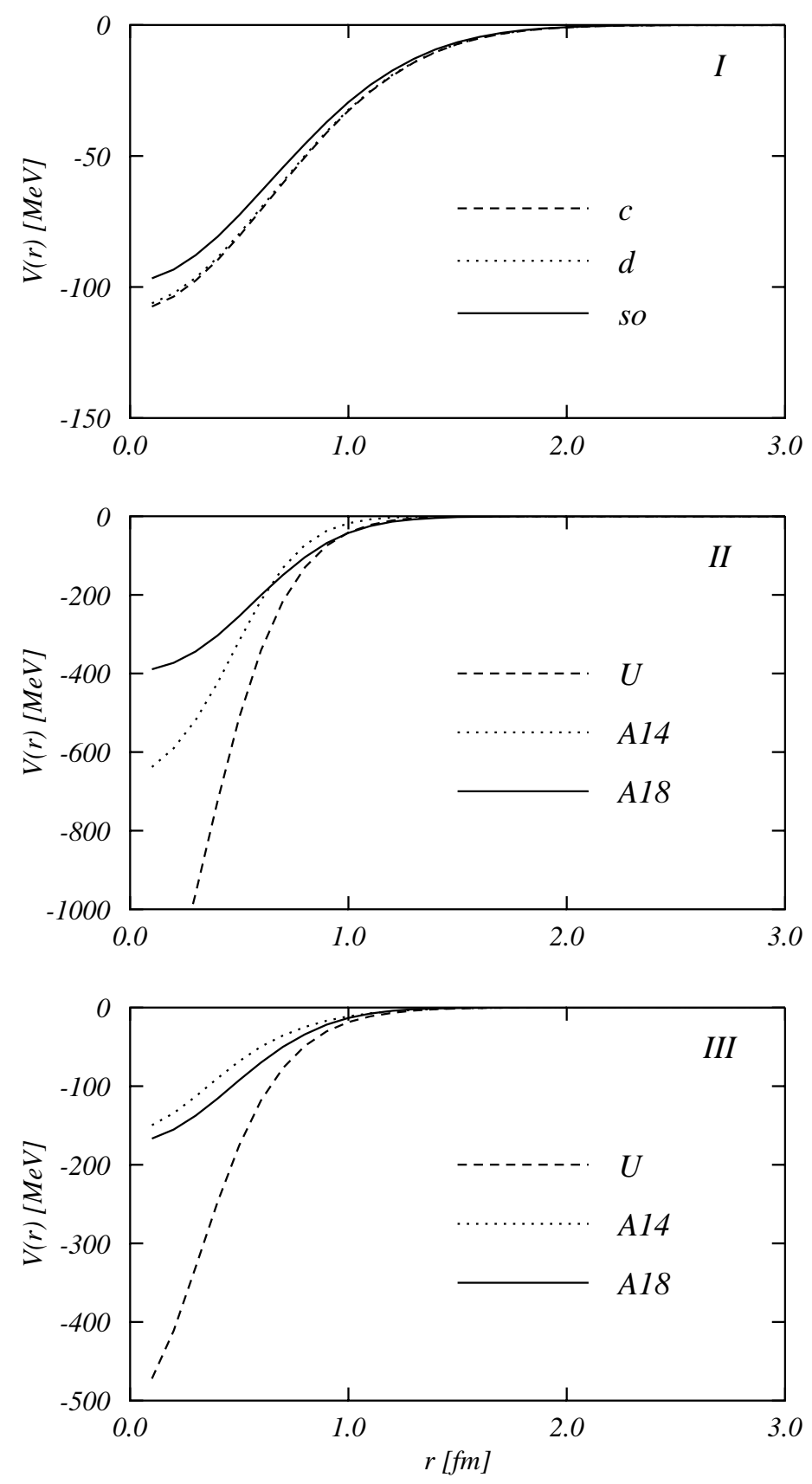

Figure 1: Radial dependence of the spin-orbit interaction used. In the panel I our gaussian interactions are shown while in the other two panels the radial dependence of the spin-orbit terms (II) and spin-orbit isospin terms (III) of the Urbana [4] Argonne V14 [5] and Argonne V18 [6] interactions. 